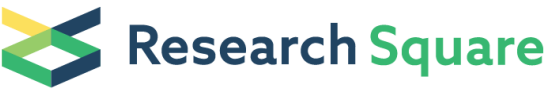 \\ Preprints are preliminary reports that have not undergone peer review. \\ They should not be considered conclusive, used to inform clinical practice, or referenced by the media as validated information.
}

\section{In-Utero Mother-to-Child SARS-CoV-2 Transmission: Viral Detection and Fetal Immune Response}

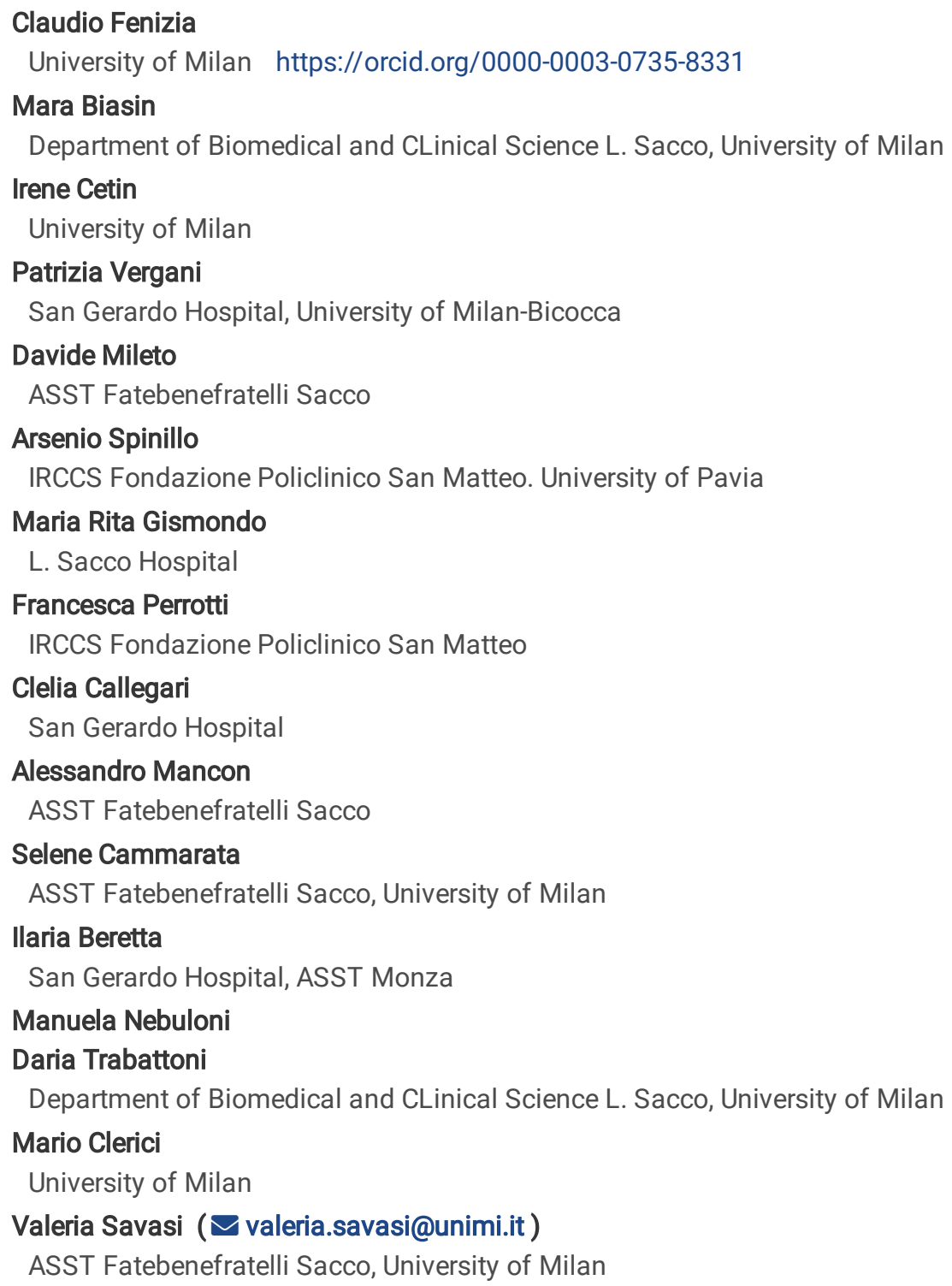

Article

Keywords: in-utero vertical transmission, SARS-CoV-2, fetal immune response, obstetric management

Posted Date: July 28th, 2020

DOI: https://doi.org/10.21203/rs.3.rs-45729/v1

License: (c) (1) This work is licensed under a Creative Commons Attribution 4.0 International License. Read Full License

Version of Record: A version of this preprint was published at Nature Communications on October 12th, 2020. See the published version at https://doi.org/10.1038/s41467-020-18933-4. 


\section{Abstract}

Pregnancy is known to increase the risk of severe illnesses in response to viral infections. Therefore, the impact of SARS-CoV-2 infection during gestational ages might be detrimental and the potential vertical transmission should be thoroughly studied.

Herein, we investigated whether SARS-CoV-2 vertical transmission is possible and, in case, whether this results in a fetal involvement. Additionally, we analyzed the role of the antibody and the inflammatory responses in placenta and plasma from SARS-CoV-2-positive pregnant women and fetuses.

31 SARS-CoV-2 pregnant women were enrolled. Real-time PCR was performed to detect the virus on maternal and newborns' nasopharyngeal swabs, vaginal swabs, maternal and umbilical cord plasma, placenta and umbilical cord biopsies, amniotic fluids and milk. Maternal and umbilical cord plasma, and milk were tested for specific anti-SARS-CoV-2 antibodies. RNA expression quantification of genes involved in the inflammatory response was performed on four selected placentas. On maternal and umbilical cord plasma of the same subjects, secreted cytokines/chemokines were quantified.

SARS-CoV-2 is found in at-term placentae and in the umbilical cord blood, in the vaginal mucosa of pregnant women and in milk. Furthermore, we report the presence of specific anti-SARS-CoV-2 IgM and IgG antibodies in the umbilical cord blood of pregnant women, as well as in milk specimens. Finally, a specific inflammatory response is triggered by SARS-CoV-2 infection in pregnant women at both systemic and placental level, and in umbilical cord blood plasma.

Our data strongly support the hypothesis that in-utero vertical transmission is possible in SARS-CoV-2 positive pregnant women. This is essential for defining proper obstetric management of COVID-19 pregnant women, or putative indications for mode and timing of delivery.

\section{Introduction}

COVID-19 pandemic is currently spreading worldwide. The number of confirmed cases currently exceeding 11.5 million people, about 590,000 deaths, and Italy representing one of the most affected countries ${ }^{1-3}$. Severe COVID-19 cases exhibit a dysfunctional immune response characterized by higher blood plasma levels of IL-1 3 , IL-2, IL-6, IL-7, IL-10, granulocyte colony- stimulating factor (G-CSF), IP-10 (CXCL10), MCP1 (CCL2), MIP1a (CCL3) and tumor necrosis factor (TNF), that mediate widespread lung inflammation and fail to successfully eradicate the pathogen ${ }^{1,4-7}$.

Maternal physiological adaptations to pregnancy are known to increase the risk of developing severe illness in response to viral infections, such as influenza; preliminary data suggest that the prognosis of SARS-CoV-2 infection could be more severe as well in pregnant women ${ }^{8}$. Vertical transmission of SARS-CoV and MERS, the two other animal coronaviruses known to infect humans, was never documented to occur. However, the number of reported cases of infected pregnant women was very low and not sufficient to draw firm conclusions (12 reported cases for SARS-CoV and 11 for MERS) ${ }^{9,10}$. Conversely, as the number of SARS-CoV-2-positive patients is rising worldwide, multiple reports focus on SARS-CoV-2-positive pregnant women ${ }^{11-16}$. No trace of the virus was detected by real-time PCR, so

$\operatorname{far}^{11,12,14,15,17}$; however, two independent manuscripts described elevated SARS-CoV-2-specific IgG and IgM antibody levels in the blood of three newborns of SARS-CoV-2 infected mothers ${ }^{18,19}$. As IgG, but not lgM, are normally transferred across the placenta, this is suggestive of in-utero infection ${ }^{18,19}$. Moreover, placental sub-membrane and cotyledon was reported positive to the virus in a 20 weeks miscarriage of a SARS-CoV-2-positive pregnant woman ${ }^{20}$.

As recently reported, the two known SARS-CoV-2 receptors Angiotensin Converting Enzyme 2 (ACE2) and Transmembrane Protease Serine 2 (TMPRSS2) are widely spread in specific cell types of the maternal-fetal interface ${ }^{21}$. Therefore, the impact of the virus on placenta and the potential of vertical transmission of SARS-CoV-2 need to be further carefully addressed.

Herein we investigated: 1) whether SARS-CoV-2 vertical transmission is possible, 2) how the production of antibodies occurs in the mother and possibly in the fetus and 3) the inflammatory profile in COVID-19-positive pregnant women and fetuses. Answering these questions is essential for understanding virus biological behavior during pregnancy, and for defining proper obstetric management of COVID-19 pregnant women.

\section{Materials And Methods}

\section{Study population}


This is a prospective multicenter study that includes 31 women: 30 with laboratory-confirmed COVID-19 infection admitted at delivery in three COVID-19 maternity hospitals of Lombardy, Italy: the 'L. Sacco' Hospital (Milan), the S. Gerardo Hospital/MBBM Foundation (Monza), and the San Matteo Hospital (Pavia) between March 9 and April 14, 2020. A further woman, (subject n. 31), the wife of Italian patient One, was found to be SARS-CoV-2-positive at the 32 gestational weeks and delivered at Buzzi maternity Hospital, a COVID-19-free hub and was admitted in the study as well.

All women underwent clinical evaluation of vital signs and symptoms, laboratory analysis and radiological chest assessment at admission at discretion of physicians. The therapeutic management was consequently tailored according to the clinical findings and national guidelines ${ }^{22}$. Demographic and anthropometric characteristics, medical and obstetric comorbidities, were recorded at enrollment through a customized data collection form. All pregnancies were singleton, with a normal course and regular checks, until delivery.

Data on mode of delivery or pregnancy termination, maternal and neonatal outcomes, and postpartum clinical evolution (e.g. breastfeeding, skin to skin, etc.) were subsequently recorded. Data accuracy was independently verified by two study investigators.

The protocol was approved by the local Medical Ethical and Institutional Review Board.

\section{Specimen collection}

Biological samples were collected at admission (T0), delivery (T1) and post-partum (T2), as summarized in figure 1. T0 samples included a nasopharyngeal swab in order to test the positivity for SARS-CoV-2. At T1, full thickness placental and umbilical cord biopsies were obtained and a $10 \mathrm{ml}$ umbilical cord blood sample was collected in EDTA after cleaning throughout the cord with a sterile gauze and physiological solution before sampling. Both biopsies and blood samples were obtained in sterile way by a dedicated operator. In case of caesarean section, if possible, amniotic fluid was collected. Moreover, a $10 \mathrm{ml}$ maternal blood sample in EDTA was collected, together with a vaginal swab before labor or cesarean section. Upon delivery, a nasopharyngeal swab was immediately performed on newborns and mothers. Approximately five days after delivery (T2), transitional breastmilk samples were collected from all breastfeeding women. For each subject, days occurring between T0 and T1 ( $\Delta \mathrm{T} 1-\mathrm{T} 0)$ were calculated. Fourteen sets of samples from the Obstetrics and Gynecology Unit of "L. Sacco" Hospital (Milan) were immediately transferred to the dedicated laboratory of Clinical Microbiology, Virology and Diagnostics, "L. Sacco" Hospital, and/or to the laboratory of Immunology, University of Milan, according to the kind of specimen, to be readily processed. Seventeen samples collected at S. Gerardo Hospital/MBBM Foundation (Monza), and San Matteo (Pavia) were frozen at $-80^{\circ} \mathrm{C}$ upon collection and transferred to the same laboratories in dry ice.

\section{Diagnostic analyses}

Molecular analysis was performed to detect viral RNA, using the automated Real-Time PCR ELITe InGenius $\AA$ system and the GeneFinderTM COVID-19 Plus RealAmp Kit assay (ELITechGroup, France). The reaction mix was prepared according to manufacturer's instruction. Three target genes, RNA-dependent RNA polymerase (RdRP), Nucleocapsid (N) and Envelope (E) have been simultaneously amplified and tested. A cycle threshold value (Ct-value) lower than 40 was defined as a positive test result according to the manufacturer's instruction.

The presence of SARS-CoV-2 specific antibodies was investigated using SARS-CoV-2 IgG and IgM chemiluminescence immunoassay (CLIA) kits on fully automated iFlash1800 analyzer (Shenzen YHLO Biotech Co., Ltd., Shenzen, China): the assay uses Nucleocapsid (N) and Spike (S) viral proteins as magnetic beads coating antigens. The value of $10.0 \mathrm{AU} / \mathrm{mL}$ was used as positivity cut-off for IgM, while 7.1 for $\operatorname{lgG}^{23}$.

\section{Tissues processing}

Placental and umbilical cord biopsies were manually dissected into few sections of approximately $2 \mathrm{~mm}^{3}$. Such sections were then thoroughly homogenized and total RNA was isolated using the acid guanidium thiocyanate-phenol-chloroform method (RNAbee, Duotech, Milan, Italy), as previously described ${ }^{24}$. Alternatively, biopsies were paraffin-embedded and stored as such.

Plasma samples were collected from blood of all the enrolled subjects, as well as plasma samples from funicular blood, amniotic fluid and vaginal swabs. Moreover, as control for molecular analyses, plasma from a SARS-CoV-2 negative pregnant woman (CTR-), as well as plasma samples from funicular blood and placental tissues were included. RNA was extracted by the Maxwell@ RSC Instrument with Maxwell $B$ RSC Viral Total Nucleic Acid Purification Kit (Promega, Fitchburg, WI, USA). As result, RNA eluted in RNAse-free water was obtained. 
Once RNA was reverse transcribed into CDNA ${ }^{24}$, real-time PCR was performed on a CFX96 (Bio-rad, CA, USA) using TaqMan probes specifically designed to target two regions of the nucleocapsid (N) gene of SARS-CoV-2. For such application, we employed the 2019-nCoV CDC qPCR Probe Assay emergency kit (IDT, lowa, USA) which include also primers and probes that target the human RNase P gene.

\section{Expression analyses of inflammatory response}

The inflammatory response was analysed in four selected RNA samples extracted from placenta biopsies of one negative control (CTR-), one SARS-CoV-2 recovered (subject n. 31) subject and two SARS-CoV-2 positive subjects (subjects $n .17$ and 25). Subjects $n .17$ and 25 gave birth to SARS-CoV-2 positive newborns, according to the first nasopharyngeal swab (T1). Analyses were performed by a PCR array that include a set of 84 optimized real-time PCR primers plus 5 housekeeping genes on a 96-well plates; the procedures suggested by the manufacturer were followed (Qiagen, Hilden, Germania). Undetermined raw CT values were set to 35 . Only variables with at least a twofold increase in their value are presented and discussed in the manuscript.

Concentration of 27 cytokines was assessed in maternal and funicular plasma samples from the very same four subjects using immunoassays formatted on magnetic beads (Bio-rad, CA, USA) according to manufacturer's protocol via Luminex 100 technology (Luminex, Texas, USA).

\section{Statistics}

For the study variables, medians and ranges were reported for quantitative variables. The analyses were performed using SPSS Statistics, Version 26.0 (IBM Corp. Armonk, NY) together with GraphPad Prism 8.

All the procedures were carried out in accordance with the GLP guidelines adopted in our laboratories.

\section{Results}

\section{Population}

Four patients were classified as severe cases, defined by the need of urgent delivery for the deterioration of maternal conditions or by ICU/sub-intensive care admission. A radiological confirmation of interstitial pneumonia was obtained on admission or antepartum for all the severe cases and in 10 (32\%) of the mild cases. Pharmacological treatment during the antepartum period of hospitalization is reported in Table 1. In the only severe case of pre-term labor (subject n. 17), corticosteroids for RDS-prophylaxis were administered. 
Baseline characteristics of the study population on admission

\begin{tabular}{|c|c|}
\hline CHARACTERISTICS AT DELIVERY & $\begin{array}{l}\text { Total study population } \\
(n=31)\end{array}$ \\
\hline \multicolumn{2}{|l|}{ MATERNAL BASELINE CHARACTERISTICS } \\
\hline Maternal age, years, median (range) & $30(15-45)$ \\
\hline RT-PCR assay of a maternal nasopharyngeal swab & $30(97)$ \\
\hline Positive n (\%) & $1 *(3)$ \\
\hline \multicolumn{2}{|l|}{ Negative $n(\%)$} \\
\hline Prepregnancy BMI, kg/m²,median (range) & $23(17-37)$ \\
\hline Known sick contact, n (\%) & $10(32)$ \\
\hline Smoking habit, n (\%) & 0 \\
\hline Ethnicity, Caucasian, n (\%) & $21(68)$ \\
\hline Chronic comorbidity, n (\%) & $11(35)$ \\
\hline Parity, nulliparous n (\%) & $14(45)$ \\
\hline Flu vaccination in pregnancy, $\mathrm{n}(\%)$ & $8(26)$ \\
\hline ANTE-PARTUM THERAPY & $10(32)$ \\
\hline Antibiotic, n (\%) & $8(26)$ \\
\hline antiviral, n (\%) & $13(42)$ \\
\hline hydroxychloroquine, n (\%) & $4(13)$ \\
\hline oxygen support without ICU admission, n (\%) & $14(45)$ \\
\hline POSITIVE CHEST X-RAY, n (\%) & $4(13)$ \\
\hline SEVERE CASE, n (\%) & $1(3)$ \\
\hline ADMISSION TO ICU, n (\%) & \\
\hline
\end{tabular}

Maternal and pregnancy outcomes in the study population are reported in Table 2. Regarding mode of delivery, three patients underwent emergency deliver for maternal respiratory indication. Among the severe cases, one needed post-partum admission to ICU and invasive ventilation for eleven days in total. 
Table 2

Maternal and pregnancy outcomes in the study population.

\begin{tabular}{|c|c|}
\hline & $\begin{array}{l}\text { Total study population } \\
\mathrm{n}=\mathbf{3 1}\end{array}$ \\
\hline Delivery mode & $25(81)$ \\
\hline - vaginal, n (\%) & $6(19)$ \\
\hline \multicolumn{2}{|l|}{ - caesarean section, $\mathrm{n}(\%)$} \\
\hline GA at delivery, weeks median (range) & $39(34.4-41.4)$ \\
\hline Induction of delivery related to COVID-19, n (\%) & $6(19)$ \\
\hline Caesarean section for severe maternal illness related COVID-19, n (\%) & $3(9)$ \\
\hline Preterm delivery, $\mathrm{n}(\%)$ & $1(3)$ \\
\hline Fetal gender, male, $\mathrm{n}(\%)$ & $18(58)$ \\
\hline Birth weight, g, median (range) & $3200(2180-4165)$ \\
\hline Umbilical artery pH, median (range) & $7.31(7.14-7.43)$ \\
\hline APGAR score $5^{\prime}<7$, n (\%) & $1(3)$ \\
\hline Newborn COVID, positive, $\mathrm{n}(\%)$ & $2(6)$ \\
\hline NICU admission, $n(\%)$ & $2(6)$ \\
\hline Skin to Skin, n (\%) & $4(13)$ \\
\hline Breastfeeding, $\mathrm{n}(\%)$ & $29(94)$ \\
\hline
\end{tabular}

Subject $\mathrm{n} .31$ became negative at week 35 of pregnancy and delivered spontaneously at week 38. Except in one case (subject n. 17), all pregnancies were full term. Subject $n$. 17 was admitted preterm at $33+6$ weeks with fever and dyspnea and delivered spontaneously at 34 +4 weeks. A female baby was born, weighing $2180 \mathrm{~g}$, with an Apgar Score of 9 and 10 at 1 and 5 minutes, respectively, with a pH of umbilical artery of 7.14. The newborn was diagnosed with COVID-19 infection through a nasopharyngeal swab and was admitted to NICU for prematurity. Subject n. 25 spontaneously delivered at week $39+2$. A male baby was born, weighing $3340 \mathrm{~g}$, with an Apgar Score of 9 and 10 at 1 and 5 minutes, respectively, and the umbilical artery pH of was 7.14. The newborn was diagnosed with SARS-CoV-2 infection through a nasopharyngeal swab upon delivery, while he tested negative $48 \mathrm{~h}$ later. Except for the two above-mentioned cases, no other newborns resulted positive to SARS-CoV-2 detection by nasopharyngeal swab. Except for two cases, all newborns were breastfed. All the newborns were healthy, including the two SARS-CoV-2 positive ones.

\section{Virus and antibody detection}

We investigated the presence of SARS-CoV-2 in the collected specimens, as shown in Table 3 and summarized in Table 4a. We detected the virus in two (6\%) maternal plasma samples (subjects $n .4$ and 17), both of them characterized by a severe clinical outcome.

Remarkably, we detected the presence of SARS-CoV-2 in vaginal swab, placental tissue and cord plasma from subject $n$. 17. Moreover, we detected SARS-CoV-2 in placental tissue from subject n. 25. We detected SARS-CoV-2 in one milk specimen only, from a severe clinical outcome case (subject n. 1). None of the tested six amniotic fluids, nor the twelve umbilical cords, resulted positive (Tables 3 and $4 a$ ). 
Table 3

Synoptic table of maternal and fetal SARS-CoV-2 virus and anti-SARS-CoV-2 antibody detection correlated to clinical data.

\begin{tabular}{|c|c|c|c|c|c|c|c|c|c|c|c|c|c|c|}
\hline \multirow[t]{2}{*}{$\begin{array}{l}\text { subject } \\
\text { n. }\end{array}$} & \multirow[t]{2}{*}{$\begin{array}{l}\text { clinical } \\
\text { outcome }\end{array}$} & \multirow{2}{*}{$\begin{array}{l}\Delta \mathrm{T} 1- \\
\text { T0 } \\
\text { (days) }\end{array}$} & \multicolumn{3}{|c|}{ maternal plasma } & \multirow{2}{*}{$\begin{array}{l}\text { vaginal } \\
\text { swab }\end{array}$} & \multirow{2}{*}{$\begin{array}{l}\text { Placenta } \\
\text { virus }\end{array}$} & \multicolumn{3}{|c|}{$\begin{array}{l}\text { Umbilical cord } \\
\text { plasma }\end{array}$} & \multirow{2}{*}{$\begin{array}{l}\text { umbilical } \\
\text { cord }\end{array}$} & \multicolumn{3}{|l|}{ milk } \\
\hline & & & virus & $\lg M$ & $\lg G$ & & & virus & $\lg M$ & $\lg G$ & & virus & $\lg M$ & IgG \\
\hline 1 & SEVERE & 2 & - & - & - & - & - & - & - & - & - & + & + & - \\
\hline 2 & MILD & 1 & - & - & - & - & - & N/A & $\mathrm{N} / \mathrm{A}$ & N/A & - & - & - & - \\
\hline 3 & MILD & 1 & - & - & - & - & - & - & - & - & - & - & - & N/A \\
\hline 4 & SEVERE & 2 & + & - & + & - & - & - & - & - & - & - & - & - \\
\hline 5 & MILD & 7 & - & - & - & - & - & - & - & - & - & N/A & N/A & N/A \\
\hline 6 & MILD & 1 & - & + & + & - & - & - & - & - & - & - & N/A & - \\
\hline 7 & MILD & 12 & - & + & + & - & - & - & - & - & - & - & - & - \\
\hline 8 & SEVERE & 6 & - & + & + & - & - & - & - & - & - & $\mathrm{N} / \mathrm{A}$ & N/A & N/A \\
\hline 9 & MILD & 1 & $\mathrm{~N} / \mathrm{A}$ & $\mathrm{N} / \mathrm{A}$ & N/A & - & - & - & - & - & - & - & - & - \\
\hline 10 & MILD & 1 & - & - & - & - & - & - & - & - & - & - & - & - \\
\hline 11 & MILD & 5 & - & - & - & - & - & - & - & - & - & - & - & - \\
\hline 12 & MILD & 4 & - & - & + & - & - & - & - & - & N/A & N/A & N/A & N/A \\
\hline 13 & MILD & 3 & - & - & + & - & - & - & - & + & - & - & - & - \\
\hline 14 & MILD & 3 & - & - & + & - & - & - & - & + & N/A & N/A & N/A & N/A \\
\hline 15 & MILD & 4 & - & - & - & - & - & - & - & - & N/A & N/A & N/A & N/A \\
\hline 16 & MILD & 2 & - & - & - & - & - & - & - & - & $\mathrm{N} / \mathrm{A}$ & $\mathrm{N} / \mathrm{A}$ & N/A & N/A \\
\hline 17 & SEVERE & 6 & + & + & + & + & + & + & - & + & $\mathrm{N} / \mathrm{A}$ & N/A & N/A & N/A \\
\hline 18 & MILD & 2 & - & - & + & - & - & - & - & + & $\mathrm{N} / \mathrm{A}$ & $\mathrm{N} / \mathrm{A}$ & N/A & N/A \\
\hline 19 & MILD & 9 & - & + & + & - & - & - & - & + & N/A & N/A & N/A & N/A \\
\hline 20 & MILD & 3 & - & + & + & - & - & - & - & + & N/A & N/A & N/A & N/A \\
\hline 21 & MILD & 13 & - & - & + & - & - & - & - & - & N/A & N/A & N/A & N/A \\
\hline 22 & MILD & 10 & - & - & - & - & - & - & - & - & $\mathrm{N} / \mathrm{A}$ & N/A & N/A & N/A \\
\hline 23 & MILD & 9 & - & - & - & - & - & - & - & - & N/A & N/A & N/A & N/A \\
\hline 24 & MILD & 12 & - & - & - & - & - & - & - & - & N/A & N/A & N/A & N/A \\
\hline 25 & MILD & 17 & - & + & + & - & + & - & + & + & N/A & N/A & N/A & N/A \\
\hline 26 & MILD & 13 & - & + & + & - & - & - & - & + & N/A & N/A & N/A & N/A \\
\hline 27 & MILD & 1 & - & - & + & - & - & - & - & + & N/A & N/A & N/A & N/A \\
\hline 28 & MILD & 3 & - & + & + & - & - & - & - & + & N/A & N/A & N/A & N/A \\
\hline 29 & MILD & 2 & - & - & + & - & - & - & - & - & $\mathrm{N} / \mathrm{A}$ & - & - & - \\
\hline 30 & MILD & 1 & - & - & + & - & - & - & - & + & $\mathrm{N} / \mathrm{A}$ & N/A & N/A & N/A \\
\hline 31 & recovered & N/A & - & - & + & N/A & - & - & - & + & N/A & N/A & N/A & N/A \\
\hline \multicolumn{15}{|c|}{ N/A = not available. } \\
\hline$\Delta \mathrm{T} 1-\mathrm{T}$ & ays) refer & & & 10 & en $t$ & & & ( $(\mathrm{TC}$ & 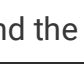 & elive & T1). & & & \\
\hline
\end{tabular}


SARS-CoV-2-specific IgM were detected in $32 \%$ of the maternal plasma, while virus-specific IgG were present in $63 \%$ of cases. Interestingly, we detected the presence of IgM in the cord plasma in one newborn only (n. 25), whose placenta tested positive to SARS-CoV-2, while IgG were present in $40 \%$ of the umbilical cord plasma. Subject n. 1 displayed IgM in milk sample (Table 3, summarized in Table $4 \mathrm{~b}$ ).

\section{Increased inflammatory response in SARS-CoV-2-positive subjects}

To determine whether SARS-CoV-2 infection results into an alteration of inflammatory gene expression in placenta tissue, we analysed the expression of 84 genes involved in the inflammatory response in four selected placenta biopsies. Results showed that placentae from SARS-CoV-2 infected patients (subjects n. 17 and 25) display a generalized immune activation profile compared to the uninfected one (CTR-) (Fig. 2). Likewise, subject 31, who was infected at gestational week 32, but fully recovered 4 weeks before delivery, showed an increased inflammatory profile when compared to CTR-. Notably, such hyper-activation status was far more evident in placenta biopsy from subject $\mathrm{n}$. 17 and even more in $\mathrm{n}$. 25, whose nasopharyngeal swab tested positive to SARS-CoV-2 detection at T1, compared to the placenta biopsy from patient 31, who tested negative at the time of delivery. The genes whose mRNA expression was clearly upregulated in subjects 17 and 25 are involved in different aspects of inflammatory response and include effector cytokines and chemokines, adaptive immunity mediators, downstream signalling molecules, Toll-Like receptors (Fig. 2).

An in-depth investigation of the cytokine/chemokine profile was carried out next in subjects CTR-, 17, 25 and 31, due to their peculiarities. A 27-cytokine multiplex assay was performed on plasma isolated from their maternal and funicular blood samples. Overall, the results obtained on maternal plasma confirmed what observed at the mRNA level in the placentae. Briefly, pro-inflammatory antiviral cytokines and chemokines were upregulated in patients $\mathrm{n}$. 17 and 25, compared to subject CTR- and 31 . Subject 25 displayed a more pronounced pro-inflammatory profile. In particular, IL-1 $\beta$ and IL-6 production were higher in subjects 25 compared to all the other subjects (Fig. 3a). The same analysis was performed on funicular plasma. As for maternal plasma, the concentration of pro-inflammatory molecules was strongly increased in the newborns from subjects n. 17 and 25. Actually, this rise was mostly evident for the chemokines: IL-8, CCL2, CCL3, CCL5 and CXCL10 (Fig. 3b).

Table 4.

Summary of maternal and fetal SARS-CoV-2 virus (a) and anti-SARS-CoV-2 antibody (b) detection.

a.

\begin{tabular}{|l|c|c|c|c|c|c|}
\hline & Maternal plasma $(\mathrm{n}=30)$ & $\begin{array}{c}\text { Vaginal swab } \\
(\mathrm{n}=31)\end{array}$ & $\begin{array}{c}\text { Placenta } \\
(\mathrm{n}=31)\end{array}$ & $\begin{array}{c}\text { Umbilical Cord plasma } \\
(\mathrm{n}=30)\end{array}$ & $\begin{array}{c}\text { Umbilical cord } \\
(\mathrm{n}=12)\end{array}$ & $\begin{array}{c}\text { Amniotic fluid } \\
(\mathrm{n}=6)\end{array}$ \\
$\begin{array}{c}\text { Milk } \\
(\mathrm{n}=11)\end{array}$ \\
\hline Pos, \% (n) & $6.7(2)$ & $3.2(1)$ & $6.4(2)$ & $3.3(1)$ & $0(0)$ & $0(0)$ \\
\hline
\end{tabular}

b.

\begin{tabular}{|c|c|c|c|}
\hline & $\begin{array}{c}\text { Maternal plasma } \\
(\mathrm{n}=30)\end{array}$ & $\begin{array}{c}\text { Umbilical cord plasma } \\
(\mathrm{n}=30)\end{array}$ & $\begin{array}{c}\text { Milk } \\
(\mathrm{n}=10)\end{array}$ \\
\hline IgM, \% (n) & $32.1(9)$ & $3.3(1)$ & $10(1)$ \\
\hline IgG, \% (n) & $63.3(19)$ & $40.0(12)$ & $0(0)$ \\
\hline IgM/IgG, \% (n) & $32.1(9)$ & $3.3(1)$ & $0(0)$ \\
\hline
\end{tabular}

\section{Discussion}

We report for the first time that SARS-CoV-2 is found in the vagina of a pregnant woman, in at-term placentae and in the umbilical cord blood. Furthermore, we report the presence of specific anti-SARS-CoV-2 IgM and IgG antibodies in the umbilical cord blood of pregnant women, as well as in milk specimens. Notably, we also provide evidences that SARS-CoV-2 can be found in milk specimen. Our data strongly support the hypothesis that in-utero vertical transmission is possible in SARS-CoV-2 positive pregnant women, consistently with 
how previously reported ${ }^{25}$. Finally, this is the first report describing the inflammatory response triggered by SARS-CoV-2 infection in pregnant women at both systemic and placental level.

Our results strongly suggest in-utero vertical transmission in 2 of the 31 (6\%) enrolled SARS-CoV-2 positive women. As one case was characterized by a severe clinical outcome (subject n.17), while the other one was classified as mild (subject n. 25), we speculate that the risk of mother-to-child viral transmission does not directly depend on the severity of disease progression. Supporting this observation, the clinical history as well as the results of the viro-immunological test performed on these two subjects were divergent. Subject $\mathrm{n}$. 17, characterized by severe conditions, was SARS-CoV-2 positive in different specimens, including maternal plasma, vagina as well as umbilical cord plasma and placenta. In this case, we hypothesize that the virus spread around the body through the bloodstream, reaching the vagina and the placenta, finally infecting the fetus. Indeed, the nasopharyngeal swab of her newborn collected upon delivery resulted positive. Notably, subject $n$. 17 was the only one to deliver prematurely at week 34 . Prematurity was indeed reported to be more frequent in SARS-CoV-2 infected patients ${ }^{8}$. We hypothesize that this might be related to the inflammatory status, as a consequence of the viral infection; alternatively, this could have been the result of a pre-existing condition that triggered the premature delivery and facilitated viral entry through the placenta. SARS-CoV-2 positivity of umbilical cord plasma from subject's 17 newborn proves an in-utero transmission. In the same woman, vagina was found positive for SARS-CoV-2. Since the presence of the virus in cord blood indicates an in-uterotransmission prior to delivery, we cannot speculate about the risk of acquiring the virus during vaginal delivery in this case. However, we cannot exclude the possibility of viral intrapartum infection when the virus is present in the vagina. Subject $\mathrm{n}$. 17 delivered 6 days after the first COVID-19 diagnosis. Probably due to the short span of $\triangle \mathrm{T} 1-\mathrm{T} 0$ time, specific anti-SARS-CoV-2 IgM were not detected in umbilical cord blood. Conversely, subject n. 25 who manifested mild symptoms was SARS-CoV-2 negative in all the biological samples analyzed (maternal plasma, vagina, umbilical cord plasma), but the placenta. However, her newborn had a SARS-CoV-2 positive nasopharyngeal swab at birth and both SARS-CoV-2 specific IgM and IgG were detected in umbilical cord plasma. Although still controversial ${ }^{26}$, the presence of anti-SARS-CoV-2 IgM strongly suggests SARS-CoV-2 infection in-utero. Of note, the positivity of the newborns' nasopharyngeal swab was not sustained over time, as the following tests were negative. We detected IgM and IgG in maternal plasma as well. This is consistent with the span of time occurred between COVID-19 diagnosis and delivery (17 days), where the median of detection of specific $\lg M / \lg G$ is 13 days $^{27}$.

We analyzed the milk collected at T2 as well. We detected the presence of SARS-CoV-2 RNA in one case only (subject n.1), who was a severe case. This is consistent with what previously reported ${ }^{28}$. However, further studies are required to assess whether this represents an infectious and replicative virus or not. Although precautions were adopted, we cannot exclude a contamination of the sample by other maternal positive sites. Moreover, we tested milk specimens for the presence of specific anti-SARS-CoV-2 IgM and IgG. We were able to detect IgM in subject n.1 only. It was previously reported that the absence of IgM and IgG in the milk is not uncommon, especially in the case of respiratory viruses ${ }^{29}$. A recent study showed the high sensitivity and specificity of iFlash automated system for antibodies detection ${ }^{23}$. However, this methodology has been adapted for detection of antibodies in milk and the sensitivity may be attenuated on this particular specimen.

Further studies are needed to ascertain long-term outcomes and potential intrauterine vertical transmission in pregnant women infected in the first or second trimester. This observation is even more relevant considering that the temporal and spatial expression of the main SARS-CoV-2 receptor, ACE2, has been reported to change significantly in maternal-fetal interface tissues in the different trimesters ${ }^{21,30}$. We can speculate on the possibility that ACE2 modulation could be directly linked to placenta susceptibility to SARS-CoV-2 infection. Alternatively, we can reason on the possibility that due to altered permeability/damages of the placenta, probably secondary to an inflammatory status, SARS-CoV-2 is able to bypass the placental barrier and reach foetal blood. This issue still remains to be addressed and further investigated.

As several lines of evidence indicate that systemic maternal infection and consequent inflammation contribute to disruption of placenta development/function and possibly favour viral vertical transmission ${ }^{31,32}$, we decided to profile the inflammatory status of four selected patients at both local (placenta) and systemic (maternal and fetal) level. Results obtained by different molecular approaches (RNA expression and protein secretion) give us the same take-home message by showing a trend of generalized immune activation in those patients (17 and 25), who were SARS-CoV-2 positive at delivery and, according to the viral-immunological analyses, infected their neonates in utero. Unexpectedly, this hyper-activation status was far more evident in SARS-CoV-2-negative biological samples (placenta biopsy, maternal and umbilical cord plasma) from subject $n$. 25 , compared to subject $n$. 17 , who displayed severe clinical condition. A plausible explanation to this apparent inconsistency stems from the observation that subjects $\mathrm{n}$. 17 was undergoing a cortisone prophylaxis during the antepartum period that could have weakened the synthesis and release of inflammatory cytokines/chemokines. Among the inflammatory factors, whose expression was evidently increased in in both placenta and cord blood samples from subjects n. 17 and 25 , 
the chemokines, CXCL10, CXCL8, CCL5, CCL3, CCL2, could have played a major role in favouring vertical transmission. Indeed, they could have created a chemotactic gradient between villi and the inter-villous space, where maternal lymphocyte circulate, thus favouring viral dissemination ${ }^{33}$. To perform such molecular analyses, only four subjects (CTR-, n. 17, n. 25 and n. 31) were chosen due to their peculiarities. However, further experiments are envisaged, in order to confirm this distinctive profile.

In conclusion, for the first time SARS-CoV-2 was detected in umbilical cord plasma, indicating that in-utero mother-to-child transmission, although rare, is possible and apparently related to a high maternal inflammatory state. Concerningly, such maternal inflammatory state is mirrored in the fetus. Although further studies are needed, this should be taken into consideration in the management of COVID-19 pregnant women.

\section{Declarations}

\section{Acknowledgements}

We would like to thank Paolo Quaini, Francesco Leone, Federica Fusè, Irma Saulle, Claudia Fusetti, Margherita Longo, Alberto Rizzo, Francesca Romeri, Federica Brunetti and Francesca Sabbatini for their support and contribution to the project. This study was supported by COVID-19 donation to Obstetrics and Gynecology and to Laboratory of Immunology, Department of Biomedical and Clinical Sciences, University of Milan, Italy. and by a grant from Flak Renewables.

\section{Authors' contributions}

VS conceived the presented idea. MB further developed the project with the help of CF. VS, IC, PV, AS, FP, CC and SC performed subject enrolment and clinical management, as well as samples collection. IB was involved in sample collection and management. CF and MB conceived, planned, performed and analysed the experiments on SARS-CoV-2 detection on plasma, biopsies and vaginal swab, and experiments on the inflammatory response. DT helped with the interpretation of the data. DM and AM conceived, planned and performed the experiments on specific antibody detection and experiments on milk, under the supervision of MG. CF, MB and VS discussed the data and wrote the manuscript. MC, IC and PV critically reviewed the manuscript. CF and MB equally contributed to the manuscript.

\section{References}

1. Huang, C. et al. Clinical features of patients infected with 2019 novel coronavirus in Wuhan, China. The Lancet 395, 497-506 (2020).

2. Grasselli, G. et al. Baseline Characteristics and Outcomes of 1591 Patients Infected With SARS-CoV-2 Admitted to ICUs of the Lombardy Region, Italy. JAMA 323, 1574-1581 (2020).

3. Dong, E., Du, H. \& Gardner, L. An interactive web-based dashboard to track COVID-19 in real time. The Lancet Infectious Diseases 20, 533-534 (2020).

4. Fu, Y., Cheng, Y. \& Wu, Y. Understanding SARS-CoV-2-Mediated Inflammatory Responses: From Mechanisms to Potential Therapeutic Tools. Virol Sin 1-6 (2020) doi:10.1007/s12250-020-00207-4.

5. Tay, M. Z., Poh, C. M., Rénia, L., MacAry, P. A. \& Ng, L. F. P. The trinity of COVID-19: immunity, inflammation and intervention. Nature Reviews Immunology 1-12 (2020) doi:10.1038/s41577-020-0311-8.

6. Qin, C. et al. Dysregulation of immune response in patients with COVID-19 in Wuhan, China. Clin Infect Dis (2020) doi:10.1093/cid/ciaa248.

7. Zhou, Y. et al. Pathogenic T cells and inflammatory monocytes incite inflammatory storm in severe COVID-19 patients. Nat/ Sci Rev doi:10.1093/nsr/nwaa041.

8. Savasi, V. et al. Clinical Findings and Disease Severity in Hospitalized Pregnant Women With Coronavirus Disease 2019 (COVID-19). Obstretrics and Gynecology.

9. Wong, S. F. et al. Pregnancy and perinatal outcomes of women with severe acute respiratory syndrome. Am J Obstet Gynecol 191, 292-297 (2004).

10. Alfaraj, S. H., Al-Tawfiq, J. A. \& Memish, Z. A. Middle East Respiratory Syndrome Coronavirus (MERS-CoV) infection during pregnancy: Report of two cases \& review of the literature. J Microbiol Immunol Infect 52, 501-503 (2019).

11. Chen, H. et al. Clinical characteristics and intrauterine vertical transmission potential of COVID-19 infection in nine pregnant women: a retrospective review of medical records. The Lancet 395, 809-815 (2020).

12. Rasmussen, S. A., Smulian, J. C., Lednicky, J. A., Wen, T. S. \& Jamieson, D. J. Coronavirus Disease 2019 (COVID-19) and pregnancy: what obstetricians need to know. Am J Obstet Gynecol (2020) doi:10.1016/j.ajog.2020.02.017.

Page $10 / 14$ 
13. Zhu, H. et al. Clinical analysis of 10 neonates born to mothers with 2019-nCoV pneumonia. Translational Pediatrics 9, 51-60-60 (2020).

14. Wang, X. et al. A case of 2019 Novel Coronavirus in a pregnant woman with preterm delivery. Clin Infect Dis (2020) doi:10.1093/cid/ciaa200.

15. Lu, Z. et al. Analysis of the pregnancy outcomes in pregnant women with COVID-19 in Hubei Province. Chinese Journal of Obstetrics and Gynecology 55, E009-E009 (2020).

16. Ferrazzi, E. et al. Vaginal delivery in SARS-CoV-2 infected pregnant women in Northern Italy: a retrospective analysis. BJOG: An International Journal of Obstetrics \& Gynaecology $\mathrm{n} / \mathrm{a}$.

17. Peng, Z. et al. Unlikely SARS-CoV-2 vertical transmission from mother to child: A case report. J Infect Public Health 13, 818-820 (2020).

18. Zeng, H. et al. Antibodies in Infants Born to Mothers With COVID-19 Pneumonia. JAMA (2020) doi:10.1001/jama.2020.4861.

19. Dong, L. et al. Possible Vertical Transmission of SARS-CoV-2 From an Infected Mother to Her Newborn. JAMA (2020) doi:10.1001/jama.2020.4621.

20. Baud, D. et al. Second-Trimester Miscarriage in a Pregnant Woman With SARS-CoV-2 Infection. JAMA (2020) doi:10.1001/jama.2020.7233.

21. Li, M., Chen, L., Zhang, J., Xiong, C. \& Li, X. The SARS-CoV-2 receptor ACE2 expression of maternal-fetal interface and fetal organs by single-cell transcriptome study. PLOS ONE 15, e0230295 (2020).

22. Clinical management of severe acute respiratory infection when COVID-19 is suspected. https://www.who.int/publicationsdetail/clinical-management-of-severe-acute-respiratory-infection-when-novel-coronavirus-(ncov)-infection-is-suspected.

23. Infantino, M. et al. Diagnostic accuracy of an automated chemiluminescent immunoassay for anti-SARS-CoV-2 IgM and IgG antibodies: an Italian experience. J. Med. Virol. (2020) doi:10.1002/jmv.25932.

24. Saulle, I. et al. Endoplasmic Reticulum Associated Aminopeptidase 2 (ERAP2) Is Released in the Secretome of Activated MDMs and Reduces in vitro HIV-1 Infection. Front. Immunol. 10, (2019).

25. Vivanti, A. J. et al. Transplacental transmission of SARS-CoV-2 infection. Nature Communications 11, 3572 (2020).

26. Kimberlin, D. W. \& Stagno, S. Can SARS-CoV-2 Infection Be Acquired In Utero?: More Definitive Evidence Is Needed. JAMA 323, 17881789 (2020).

27. Long, Q.-X. et al. Antibody responses to SARS-CoV-2 in patients with COVID-19. Nature Medicine 1-4 (2020) doi:10.1038/s41591-0200897-1.

28. Groß, R. et al. Detection of SARS-CoV-2 in human breastmilk. The Lancet 395, 1757-1758 (2020).

29. Dixon, D.-L. The Role of Human Milk Immunomodulators in Protecting Against Viral Bronchiolitis and Development of Chronic Wheezing Illness. Children (Basel) 2, 289-304 (2015).

30. Valdés, G. et al. Distribution of Angiotensin-(1-7) and ACE2 in Human Placentas of Normal and Pathological Pregnancies. Placenta 27, 200-207 (2006).

31. Lee, J. K., Oh, S.-J., Park, H. \& Shin, O. S. Recent Updates on Research Models and Tools to Study Virus-Host Interactions at the Placenta. Viruses 12, 5 (2020).

32. Heerema-McKenney, A. Defense and infection of the human placenta. APMIS 126, 570-588 (2018).

33. Kim, C. J., Romero, R., Chaemsaithong, P. \& Kim, J.-S. Chronic Inflammation of the Placenta: Definition, Classification, Pathogenesis, and Clinical Significance. Am J Obstet Gynecol 213, S53-S69 (2015).

\section{Figures}




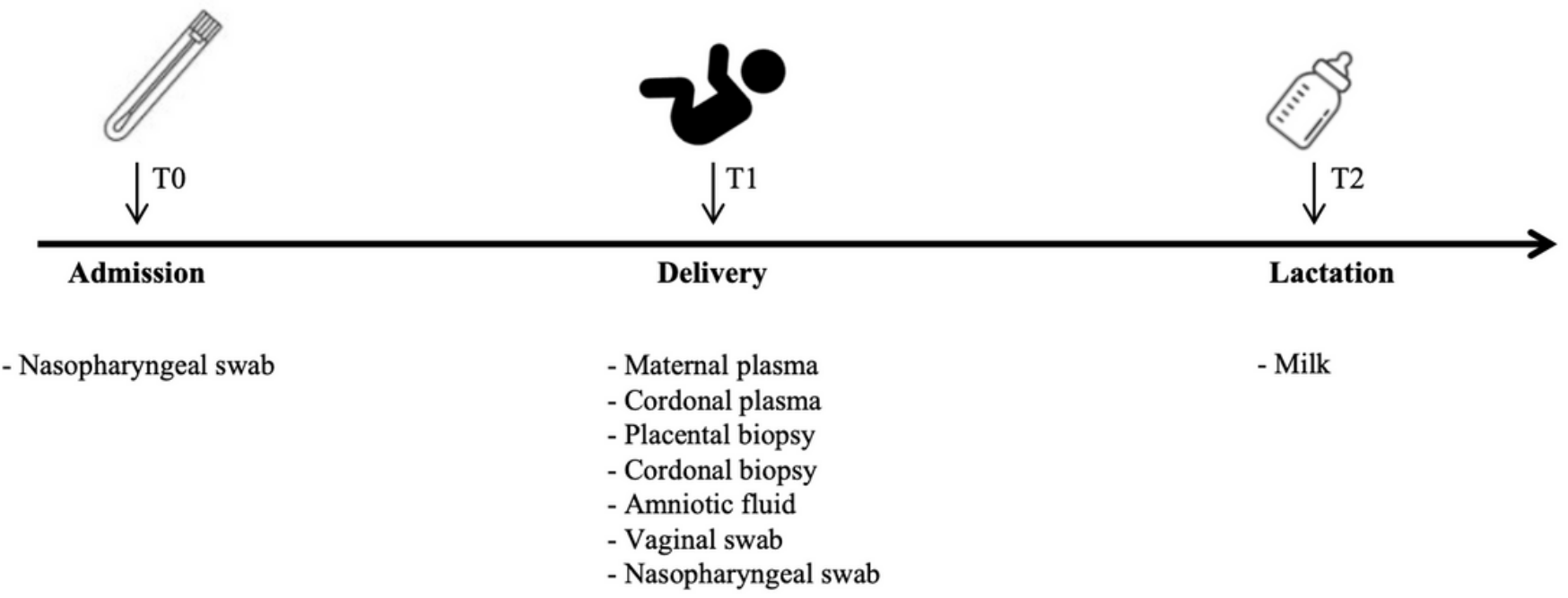

Figure 1

Specimen collection timeline. List of specimens collected at admission to the study (T0), at delivery (T1) and during lactation (T2) for each enrolled subject. 

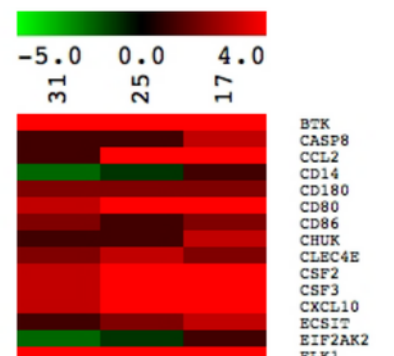

\begin{tabular}{|c|c|c|c|c|}
\hline & Gene & $\mathrm{N}^{\circ} 31$ & $N^{\circ} 25$ & $\mathbf{N}^{\circ} 17$ \\
\hline \multirow{21}{*}{ 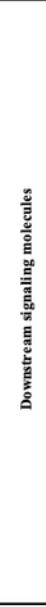 } & Fos & $-2 \cdot 41$ & 1.93 & 4.32 \\
\hline & ELK1 & $3 \cdot 61$ & 4.74 & $4 \cdot 41$ \\
\hline & IRAK1 & $1 \cdot 47$ & 2.63 & 2.08 \\
\hline & IRAK2 & $3 \cdot 16$ & 4.77 & 3.34 \\
\hline & IRF3 & $-3 \cdot 71$ & -2.59 & -1.92 \\
\hline & JUN & $-1 \cdot 11$ & $4 \cdot 30$ & 30.27 \\
\hline & MAP2K3 & $-3 \cdot 03$ & $-1 \cdot 14$ & $2 \cdot 41$ \\
\hline & MAPKSIP3 & 3.27 & $9 \cdot 16$ & 6.02 \\
\hline & NFKB2 & 1.04 & 1.25 & $4 \cdot 20$ \\
\hline & NFKBIA & -4.86 & 1.56 & 7.36 \\
\hline & \begin{tabular}{|l} 
NFKBIL1 \\
\end{tabular} & 1.67 & 2.52 & $2 \cdot 48$ \\
\hline & PELII & $-1 \cdot 04$ & $2 \cdot 26$ & 4.03 \\
\hline & PPARA & 2.39 & 5.52 & 2.97 \\
\hline & PRKRA & $-2 \cdot 97$ & 1.03 & $2 \cdot 33$ \\
\hline & SARMI & 2.58 & 1.5 & 3.51 \\
\hline & SIGIRR & 2.75 & 3.19 & 3.14 \\
\hline & TBKI & -2.87 & $-3 \cdot 42$ & -1.71 \\
\hline & TICAMI & $1 \cdot 16$ & 1.31 & 4.35 \\
\hline & TIRAP & 2.07 & $4 \cdot 16$ & 2.75 \\
\hline & TOLIP & 2.97 & 2.39 & 2.73 \\
\hline & TRAF6 & 3.58 & $3 \cdot 26$ & 2.38 \\
\hline \multirow{15}{*}{ 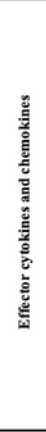 } & $\mathrm{CCL}_{2}$ & 1.44 & 4.58 & 3.61 \\
\hline & CSF2 & 2.87 & 3.80 & 4.86 \\
\hline & CSF3 & 2.62 & 3.69 & $9 \cdot 32$ \\
\hline & CXCL10 & 3.14 & $7 \cdot 14$ & 7.36 \\
\hline & IFNB1 & 2.68 & 7.34 & 3.36 \\
\hline & IFNG & 1.14 & 1.65 & 3.07 \\
\hline & L10 & $2 \cdot 17$ & 3.82 & 3.51 \\
\hline & L12A & 2.51 & $2 \cdot 94$ & 1.91 \\
\hline & L1A & 1.39 & 2.82 & $-1 \cdot 06$ \\
\hline & IL1B & $-1 \cdot 39$ & 2.09 & 1.66 \\
\hline & 世.2 & 1.56 & 2.78 & 2.81 \\
\hline & 世6 & 1.72 & 16.51 & 8.06 \\
\hline & CXCL8 & 1.24 & 33.24 & 19.84 \\
\hline & LTA & 3.86 & $11 \cdot 20$ & $3 \cdot 34$ \\
\hline & TNF & 5.70 & 3.49 & $10 \cdot 70$ \\
\hline \multirow{3}{*}{ 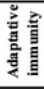 } & CD80 & 2.89 & 3.59 & 7.01 \\
\hline & CD86 & 1.82 & 1.27 & $2 \cdot 11$ \\
\hline & BTK & $16 \cdot 00$ & 7.65 & 9.65 \\
\hline \multirow{8}{*}{ 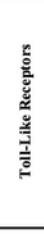 } & TLR1 & 1.65 & 3.28 & $1 \cdot 48$ \\
\hline & TLR10 & $5 \cdot 46$ & 5.84 & 9.19 \\
\hline & TLR2 & 3.97 & 2.01 & 1.64 \\
\hline & TLR3 & $-1 \cdot 23$ & $-4 \cdot 30$ & $-2 \cdot 27$ \\
\hline & TLR6 & $3 \cdot 84$ & $4 \cdot 01$ & 2.25 \\
\hline & TLR7 & 1.71 & 1.40 & $-3 \cdot 78$ \\
\hline & TLR8 & $12 \cdot 21$ & 6.21 & $2 \cdot 00$ \\
\hline & TLR9 & $12 \cdot 47$ & 7.24 & 3.63 \\
\hline \multirow{4}{*}{ 这 } & CLECAE & 2.13 & 2.69 & 1.78 \\
\hline & HSPAIA & -1.82 & 1.60 & $4 \cdot 20$ \\
\hline & HSPD1 & -6.19 & -2.98 & -6.06 \\
\hline & LY86 & 1.43 & $4 \cdot 30$ & 2.89 \\
\hline
\end{tabular}

\section{Figure 2}

mRNA expression of 84 genes that are part of the inflammatory response is altered in placentae from subjects who experienced SARSCoV-2 in-utero viral transmission. Real-time PCR array was performed on placenta biopsies from subjects n. 31,25 and 17. Results are shown as a ratio of each SARS-CoV-2-positive subject compared to a SARS-CoV-2-negative one (CTR-). Gene expression (nfold) is shown as a color scale from green to red (MEV multiple experiment viewer software). Only targets showing at least $>2$-fold modulation are shown in the table. 

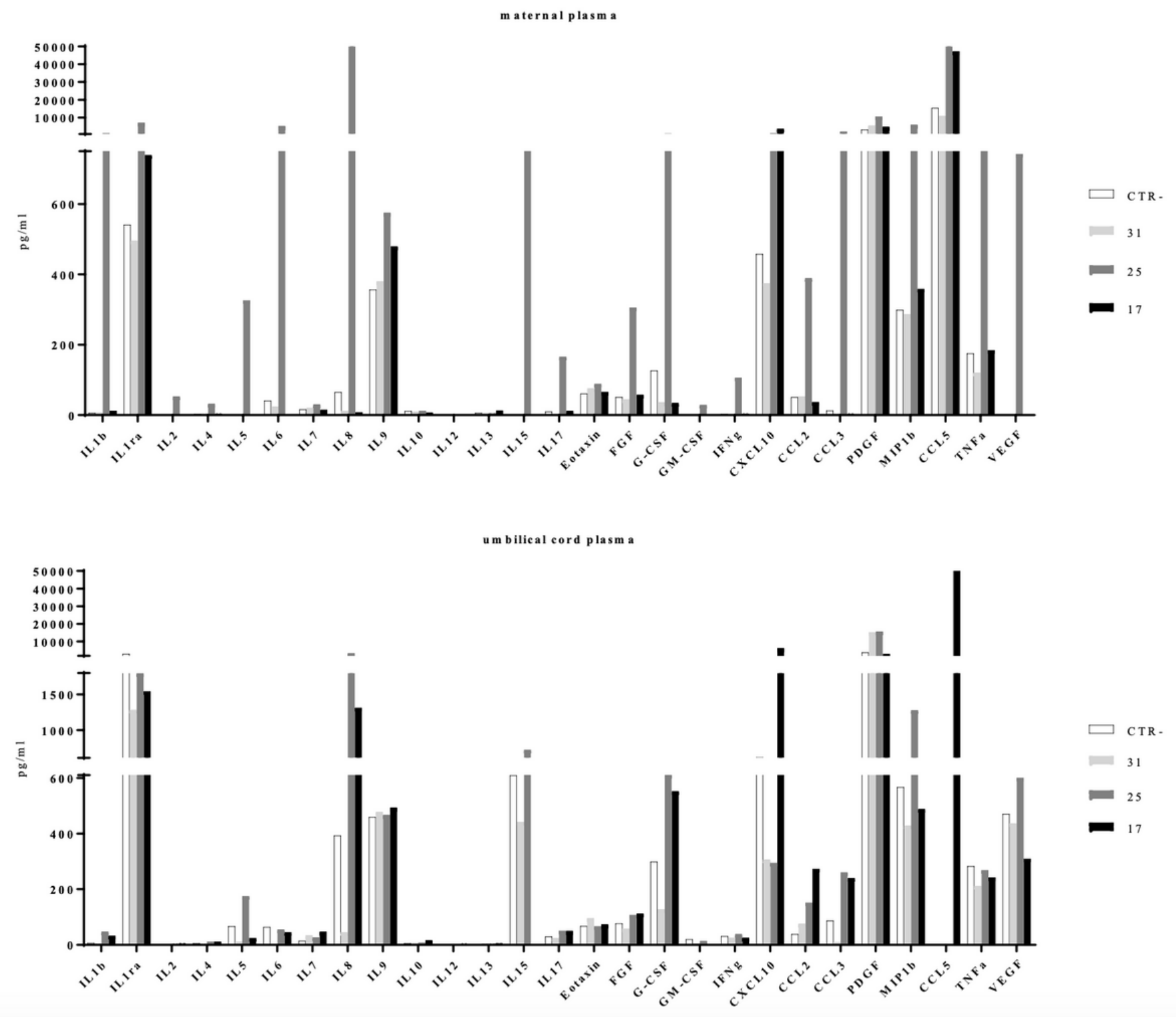

Figure 3

Protein secretion of 27 cytokines/chemokines that are part of the inflammatory response is altered in maternal and umbilical cord plasma from subjects who experienced SARS-CoV-2 in-utero viral transmission. Multiplex array was performed on maternal and umbilical plasma from subjects n. 31,25 and 17. As reference, a SARS-CoV-2-negative plasma is shown (CTR-). Protein concentration is shown as pg/ml of plasma. 